\title{
SPECTRAL ANALYSIS OF 3-(ADAMANTAN-1-YL)-4-ETHYL-1-[(4-PHENYLPIPERAZIN-1-YL) METHYL]-1H-1,2,4-TRIAZOLE-5(4H)-THIONE
}

\author{
Y. L. Mindarava, ${ }^{a}$ M. B. Shundalau, ${ }^{\text {a,b* }}$ L. H. Al-Wahaibi, \\ A. A. El-Emam, ${ }^{\text {d }}$ A. S. Matsukovich, ${ }^{\mathrm{e}}$ and S. V. Gaponenko ${ }^{\mathrm{a}, \mathrm{e}}$
}

UDC 539.19;535.34;535.375.5

Vibrational IR (3200-650 $\left.\mathrm{cm}^{-1}\right)$ and Raman spectra $\left(3200-150 \mathrm{~cm}^{-1}\right)$ of adamantane-containing 3-(adamantan1-yl)-4-ethyl-1-[(4-phenylpiperazin-1-yl)methyl]-1H-1,2,4-triazole-5(4H)-thione, which is promising for drug design, were examined. The UV/Vis spectrum (450-200 nm) of the compound in EtOH was measured. Full geometry optimization using density functional theory (DFT) in the B3LYP/cc-pVDZ approximation allowed the equilibrium configuration of the molecule to be determined and IR and Raman spectra to be calculated. Based on these, the experimental vibrational IR and Raman spectra were interpreted and the biological activity indices were predicted. The UV/Vis spectrum of the title compound was simulated at the time-dependent DFT/CAM-B3LYP/cc-pVDZ level with and without solvent effects and at the ab initio multi-reference perturbation theory XMCQDPT2 level. The $U V / V i s$ spectrum that was simulated using the multi-reference XMCQDPT2 approximation agreed very successfully with the experimental data, in contrast to the single-reference DFT method. This was probably a consequence of intramolecular charge transfer.

Keywords: 3-(adamantan-1-yl)-4-ethyl-1-[(4-phenylpiperazin-1-yl)methyl]-1H-1,2,4-triazole-5(4H)-thione, FTIR spectrum, Raman spectrum, UV/Vis spectrum, density functional theory, multi-reference perturbation theory, biological activity.

Introduction. Diamondoids (including adamantane as the simplest representative of the family) and their derivatives are viable candidates for drug development and delivery systems [1-3]. An adamantane motif in organic compounds increases their biological activity by increasing the lipophilicity and facilitating more efficient transport across biological membranes. Adamantane derivatives exhibit antiviral activity against influenza virus [4-6] and HIV [7-9] and possess antimicrobial [10-15], anti-inflammatory [15-17], and antiproliferative activity [18].

The present work studied the spectral characteristics of 3-(adamantan-1-yl)-4-ethyl-1-[(4-phenylpiperazin-1-yl) methyl]-1H-1,2,4-triazole-5(4H)-thione $\left(\mathrm{C}_{25} \mathrm{H}_{35} \mathrm{~N}_{5} \mathrm{~S}\right)$ using experimental spectral (vibrational and electronic absorption) and theoretical methods (density functional theory, ab initio multi-reference perturbation theory). The adamantane derivative was recently synthesized and characterized by a single-crystal x-ray structure analysis [19].

Previously, quantum-chemical calculations determined the spectral characteristics of several adamantane derivatives of interest for drug synthesis, i.e., $N^{\prime}$-(adamantan-2-ylidene)benzohydrazide [20, 21], 3-(adamantan-1-yl)-1-[(4-benzylpiperazin1-yl)methyl]-4-phenyl-1H-1,2,4-triazole-5(4H)-thione [22], and 3-(adamantan-1-yl)-4-phenyl-1-[(4-phenylpiperazin-1-yl) methyl]-1H-1,2,4-triazole-5(4H)-thione [22]. The results of the present research may be useful in medicinal chemistry for new drug discovery owing to the interest in the pharmacological and structural properties of adamantane derivatives.

Experimental. IR absorption spectra $\left(3200-650 \mathrm{~cm}^{-1}\right)$ were recorded using a Nexus FT-IR spectrometer (Thermo

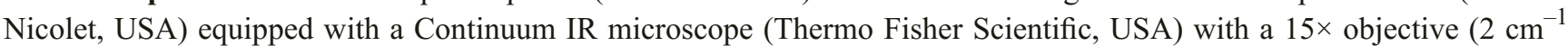

*To whom correspondence should be addressed.

${ }^{a}$ Belarusian State University, 4 Nezavisimost' Ave., Minsk, 220030, Belarus; email: shundalov@bsu.by; ${ }^{\mathrm{b}}$ A. N. Sevchenko Institute of Applied Physical Problems, Belarusian State University, Minsk, Belarus; ${ }^{\mathrm{c}}$ College of Sciences, Princess Nourah Bint Abdulrahman University, Riyadh 11671, Saudi Arabia; ${ }^{\mathrm{d}}$ College of Pharmacy, King Saud University,

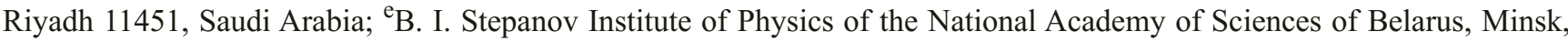
Belarus. Translated from Zhurnal Prikladnoi Spektroskopii, Vol. 85, No. 2, pp. 181-193, March-April, 2018. Original article submitted October 27, 2017. 
resolution). Samples were prepared by grinding crystals into a powder that was spread into a thin film on the reflecting surface of the substrate (Al foil). The obtained signal was both scattered radiation from the sample and radiation that passed through the sample and was reflected from the substrate surface. Raman spectra $\left(3200-150 \mathrm{~cm}^{-1}\right)$ were recorded using a continuous DPSS laser $(\lambda=532 \mathrm{~nm}$, power $7 \mathrm{~mW})$ as the excitation source. The angle between the excitation and recorded signal directions was $180^{\circ}$. The recording system consisted of a Solar TII S3901 spectrograph equipped with a diffraction grating (1200 lines $/ \mathrm{mm}$ ) and CCD array (Princeton Instruments) cooled by liquid $\mathrm{N}_{2}$. The signal accumulation time was $60 \mathrm{~s}$. Electronic absorption spectra (450-200 nm) of the compound in EtOH were recorded using a Cary 500 spectrophotometer. The solvent effect was minimized by placing a cuvette with EtOH in the reference channel.

Structures and vibrational spectra of the compound were calculated using the GAMESS-US quantum-chemical suite [23]. Results were illustrated using MacMolPlt [24] and ORTEP [25] programs. Density functional theory (DFT) with a Dunning correlation-consistent, polarized valence, double-zeta basis set [26] and hybrid exchange-correlated functional B3LYP [27-29] was used to optimize the equilibrium structure and to calculate the Hessian (force field), eigenfrequencies of modes in the harmonic approximation, and IR and Raman intensities.

Calculations in the first stage searched for a stable configuration using gradient optimization of all molecular geometric parameters. When the optimization was completed, the eigenfrequencies of modes in the harmonic approximation and intensities in IR and Raman vibrational spectra were calculated. The force field was computed considering two shifts (in positive and negative directions) of each atom relative to its equilibrium position along each of three Cartesian axes. This approach improved by $20-50 \mathrm{~cm}^{-1}$ (or $\sim 1 \%$ ) the accuracy of the calculated vibrational frequencies. In several instances, imaginary frequencies for low-frequency bending vibrations were avoided. The aforementioned improvement in the accuracy turned out to be very important because the harmonic frequencies of stretching modes of terminal groups that are calculated using the B3LYP/cc-pVDZ approximation are usually $4-5 \%$ greater than the experimental ones. The lack of imaginary frequencies in the calculated spectrum confirmed that the obtained structure was located at a minimum on the potential-energy surface. Vibrational bands and lines were assigned by calculating the potential-energy distribution over internal coordinates.

Time-dependent density functional theory (TDDFT) [30] is currently widely used to simulate electronic spectra of polyatomic molecular systems. Quantum-chemical calculations using the TDDFT formalism with the local spin density approximated by gradient corrections (i.e., using standard exchange-correlation functionals, e.g., hybrid functional B3LYP) are known to give deviations up to $0.4 \mathrm{eV}$ for the energies of the lower excited states from the experimental ones [31]. This is especially typical of molecular systems with charge transfer. Phenyl, piperazine, and triazole groups in the studied molecule allowed it to be classified as a molecular system that could exhibit intramolecular charge transfer (ICT). The Coulomb-attenuating method (CAM) approximation (or refined LRC method) that was developed for such instances [32] could sometimes improve the qualitative and quantitative calculations. Thus, the spectral and energy characteristics of the excited singlet states of the studied compound were calculated using the TDDFT method with cc-pVDZ basis set [26] and hybrid functional CAM-B3LYP [32]. The solvent (EtOH) effect was accounted for in the solvation model density (SMD) approximation [33]. The quantum-chemical GAMESS-US suite was also used for the calculations.

Multi-reference approximations (e.g., the $a b$ initio multi-reference perturbation theory method) are alternatives to the TDDFT method. Our recent work [21] demonstrated that ab initio calculations in the multi-reference approximation CASSCF/XMCQDPT2 [34] explained very successfully the electronic absorption spectrum of $N^{\prime}$-(adamantan-2-ylidene) benzohydrazide considering the presence of four conformers in solution [21].

The electronic absorption spectrum of the studied molecule was calculated in the CASSCF/XMCQDPT2 approximation using the Firefly quantum-chemical suite [35] and standard basis set cc-pVDZ [26]. The first stage included CASSCF calculations with the density-matrix averaged over seven states (four singlets and three triplets) for two active electrons involving four active orbitals. Then, calculations used the XMCQDPT2 approximation [34]. The electronic correlation energy for all 117 doubly occupied orbitals in the ground configuration was taken into account. The energy denominator shift (EDS) was 0.01 .

The structure determination of the studied molecule enabled calculation of its biological activity indices (probability to be active/inactive, $\mathrm{Pa} / \mathrm{Pi}$ ), i.e., the probability that biological activity of a particular type was present (absent). The biological activities were obtained using the PASS database [36], the online version of which [37] predicted greater than 4,000 different biological activities based only on the compound structural data.

Results and Discussion. Figure 1 shows the equilibrium structure of the studied molecule. The molecule of 3-(adamantan-1-yl)-4-ethyl-1-[(4-phenylpiperazin-1-yl)methyl]-1H-1,2,4-triazole-5(4H)-thione contained the functional groups adamantyl $(\mathrm{A})$, phenyl $(\mathrm{Ph})$, piperazine $(\mathrm{P})$, and triazole rings $(\mathrm{T})$ and methyl $(\mathrm{M})$ and ethyl $(\mathrm{Et})$. Several of them 


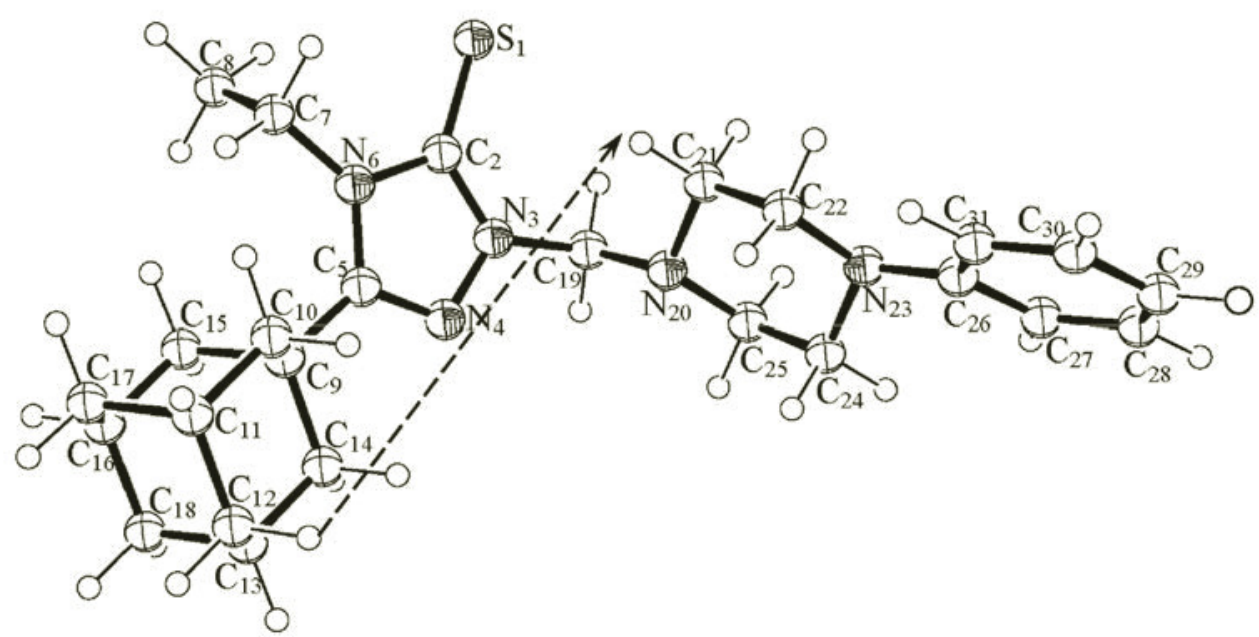

Fig. 1. Equilibrium structure and electric dipole moment of 3-(adamantan-1-yl)-4-ethyl-1[(4-phenylpiperazin-1-yl)methyl]-1H-1,2,4-triazole-5(4H)-thione calculated in the B3LYP/cc-pVDZ approximation.

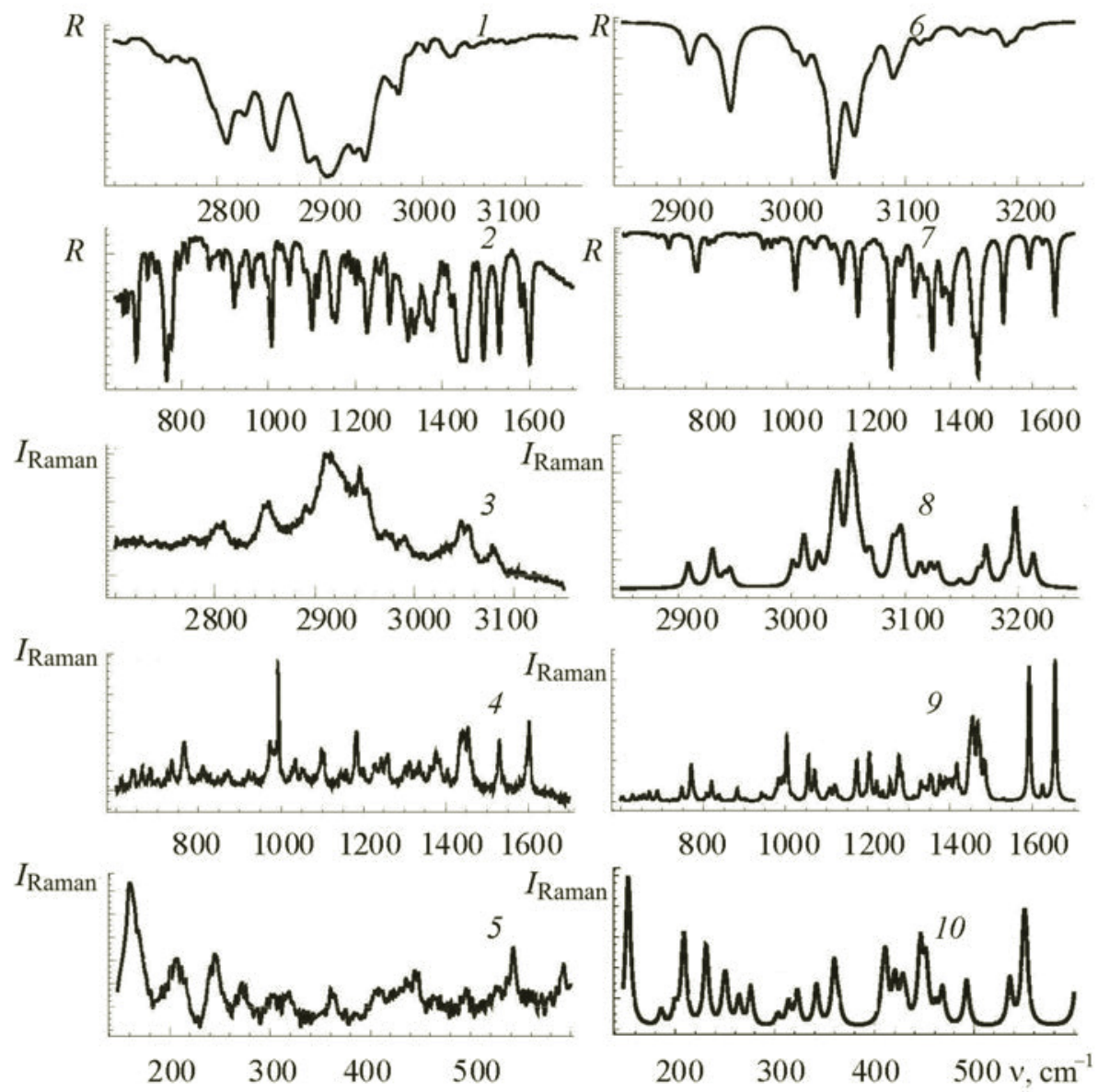

Fig. 2. Experimental (1-5) and calculated (B3LYP/cc-pVDZ) (6-10) IR (1, 2, 6, 7) and Raman spectra (3-5, 8-10) of 3-(adamantan-1-yl)-4-ethyl-1-[(4-phenylpiperazin-1-yl) methyl]-1H-1,2,4-triazole-5(4H)-thione. 
TABLE 1. Frequencies $\left(\mathrm{cm}^{-1}\right)$ and Intensities in Experimental and Calculated IR and Raman Spectra of 3-(Adamantan-1-yl)-4ethyl-1-[(4-phenylpiperazin-1-yl)methyl]-1H-1,2,4-triazole-5(4H)-thione

\begin{tabular}{|c|c|c|c|c|c|}
\hline$v_{\mathrm{IR}}^{\exp }$ & $v_{\text {Raman }}^{\exp }$ & $v^{\text {calc }}$ & $I_{\mathrm{IR}}^{\mathrm{calc}}$ & $I_{\text {Raman }}^{\text {calc }}$ & Assignment \\
\hline & $3080 \mathrm{w}$ & 3213 & 0.04 & 0.34 & $v \mathrm{CH}(\mathrm{Ph})$ \\
\hline & $3055 \mathrm{~m}$ & 3197 & 0.10 & 0.85 & $v \mathrm{CH}(\mathrm{Ph})$ \\
\hline 3047 vw & $3047 \mathrm{~m}$ & 3190 & 0.17 & 0.15 & $v \mathrm{CH}(\mathrm{Ph})$ \\
\hline $3028 \mathrm{w}$ & & 3172 & 0.06 & 0.42 & $v \mathrm{CH}(\mathrm{Ph})$ \\
\hline $3003 \mathrm{vw}$ & & 3165 & 0.04 & 0.15 & $v \mathrm{CH}(\mathrm{Ph})$ \\
\hline $2986 \mathrm{sh}$ & $2989 \mathrm{w}$ & & & & $2 \times 1495$ \\
\hline $2977 \mathrm{~m}$ & & 3149 & 0.09 & 0.07 & $v \mathrm{CH}_{3}+v \mathrm{CH}_{2}(\mathrm{Et})$ \\
\hline \multirow[t]{8}{*}{$2971 \mathrm{~m}$} & $2970 \mathrm{w}$ & 3129 & 0.00 & 0.23 & $v \mathrm{CH}_{3}+v \mathrm{CH}_{2}(\mathrm{Et})$ \\
\hline & & 3122 & 0.09 & 0.22 & $v \mathrm{CH}(\mathrm{P})$ \\
\hline & & 3113 & 0.12 & 0.24 & $v \mathrm{CH}_{3}$ \\
\hline & $2953 \mathrm{~s}$ & 3098 & 0.08 & 0.17 & $v \mathrm{CH}_{2}(\mathrm{M})$ \\
\hline & & 3096 & 0.06 & 0.38 & $v \mathrm{CH}(\mathrm{P})$ \\
\hline & & 3093 & 0.09 & 0.21 & $v \mathrm{CH}(\mathrm{P})$ \\
\hline & & 3089 & 0.15 & 0.26 & $v \mathrm{CH}_{2}(\mathrm{~A})$ \\
\hline & & 3088 & 0.19 & 0.14 & $v \mathrm{CH}_{2}(\mathrm{~A})$ \\
\hline 2943 vs & 2941 vs & 3070 & 0.13 & 0.20 & $v \mathrm{CH}_{2}(\mathrm{~A})$ \\
\hline \multirow[t]{6}{*}{2934 vs } & & 3068 & 0.08 & 0.17 & $v \mathrm{CH}_{2}(\mathrm{~A})+v \mathrm{CH}_{2}(\mathrm{M})$ \\
\hline & & 3061 & 0.17 & 0.27 & $v \mathrm{CH}(\mathrm{P})$ \\
\hline & & 3056 & 0.56 & 0.63 & $v \mathrm{CH}_{2}(\mathrm{~A})$ \\
\hline & 2917 vs & 3052 & 0.20 & 1.00 & $v \mathrm{CH}_{2}(\mathrm{~A})+v \mathrm{CH}(\mathrm{A})$ \\
\hline & & 3051 & 0.10 & 0.13 & $v \mathrm{CH}_{2}(\mathrm{~A})$ \\
\hline & & 3049 & 0.04 & 0.24 & $v \mathrm{CH}_{2}(\mathrm{~A})$ \\
\hline \multirow[t]{2}{*}{2911 vs } & & 3041 & 0.32 & 0.79 & $v \mathrm{CH}_{2}(\mathrm{~A})+v \mathrm{CH}(\mathrm{A})$ \\
\hline & & 3038 & 0.19 & 0.41 & $v \mathrm{CH}_{3}(\mathrm{M})$ \\
\hline \multirow[t]{3}{*}{2907 vs } & 2909 vs & 3037 & 0.55 & 0.15 & $v \mathrm{CH}_{2}(\mathrm{~A})+v \mathrm{CH}(\mathrm{A})$ \\
\hline & & 3035 & 0.37 & 0.23 & $v \mathrm{CH}_{2}(\mathrm{~A})+v \mathrm{CH}(\mathrm{A})$ \\
\hline & & 3025 & 0.19 & 0.09 & $v \mathrm{CH}_{2}(\mathrm{~A})+v \mathrm{CH}(\mathrm{A})$ \\
\hline \multirow[t]{2}{*}{2888 vs } & $2889 \mathrm{~m}$ & 3023 & 0.02 & 0.21 & $v \mathrm{CH}_{2}(\mathrm{~A})+v \mathrm{CH}(\mathrm{A})$ \\
\hline & & 3012 & 0.10 & 0.19 & $v \mathrm{CH}_{2}(\mathrm{~A})+v \mathrm{CH}(\mathrm{A})$ \\
\hline \multirow[t]{4}{*}{$2853 \mathrm{~s}$} & $2855 \mathrm{~m}$ & 3011 & 0.09 & 0.16 & $v \mathrm{CH}_{2}(\mathrm{~A})+v \mathrm{CH}(\mathrm{A})$ \\
\hline & $2847 \mathrm{~m}$ & 3010 & 0.07 & 0.20 & $v \mathrm{CH}_{2}(\mathrm{~A})+v \mathrm{CH}(\mathrm{A})$ \\
\hline & & 3001 & 0.14 & 0.25 & $v \mathrm{CH}_{2}(\mathrm{M})$ \\
\hline & & 2945 & 0.66 & 0.19 & $v \mathrm{CH}(\mathrm{P})+v \mathrm{CH}_{2}(\mathrm{P})$ \\
\hline \multirow[t]{3}{*}{$2826 \mathrm{~m}$} & & 2940 & 0.21 & 0.10 & $v \mathrm{CH}(\mathrm{P})+v \mathrm{CH}_{2}(\mathrm{P})$ \\
\hline & & 2930 & 0.08 & 0.40 & $v \mathrm{CH}(\mathrm{P})+v \mathrm{CH}_{2}(\mathrm{P})$ \\
\hline & & 2909 & 0.34 & 0.27 & $v \mathrm{CH}(\mathrm{P})$ \\
\hline $2809 \mathrm{~s}$ & $2809 \mathrm{w}$ & & & & $1450+1362$ \\
\hline \multirow[t]{3}{*}{$2795 \mathrm{sh}$} & $2802 \mathrm{w}$ & & & & $1441+1361$ \\
\hline & & & & & $1418+1377$ \\
\hline & $2776 \mathrm{vw}$ & & & & $1404+1374$ \\
\hline $2769 w$ & & & & & $1579+1190$ \\
\hline $2751 \mathrm{w}$ & & & & & $1472+1279$ \\
\hline
\end{tabular}


TABLE 1. (Continued)

\begin{tabular}{|c|c|c|c|c|c|}
\hline$v_{\mathrm{IR}}^{\exp }$ & $v_{\text {Raman }}^{\exp }$ & $v^{\text {calc }}$ & $I_{\mathrm{IR}}^{\text {calc }}$ & $I_{\text {Raman }}^{\text {calc }}$ & Assignment \\
\hline 1599 vs & $1602 \mathrm{~s}$ & 1655 & 0.63 & 0.28 & $v C C(\mathrm{Ph})+\delta$ (i.p. $) \mathrm{CCC}(\mathrm{Ph})$ \\
\hline $1579 \mathrm{~m}$ & & 1624 & 0.06 & 0.03 & $v C C(P h)+\delta($ i.p. $) C C C(P h)$ \\
\hline 1533 vs & $1529 \mathrm{~m}$ & 1592 & 0.26 & 0.27 & $v \mathrm{CN}(\mathrm{T})$ \\
\hline 1495 vs & & 1529 & 0.66 & 0.00 & $v \mathrm{CC}(\mathrm{Ph})+\delta($ i.p. $) \mathrm{CCC}(\mathrm{Ph})$ \\
\hline \multirow[t]{2}{*}{$1472 \mathrm{w}$} & & 1493 & 0.01 & 0.01 & $\sigma \mathrm{CH}_{2}(\mathrm{~A})$ \\
\hline & & 1486 & 0.04 & 0.05 & $\sigma \mathrm{CH}_{2}(\mathrm{P})+\sigma \mathrm{CH}_{2}(\mathrm{M})$ \\
\hline \multirow[t]{3}{*}{1450 vs } & $1453 \mathrm{~m}$ & 1483 & 0.00 & 0.01 & $v C C(\mathrm{Ph})+\delta($ i.p. $) C C C(\mathrm{Ph})$ \\
\hline & & 1482 & 0.02 & 0.02 & $\sigma \mathrm{CH}_{2}(\mathrm{~A})+\sigma \mathrm{CH}_{2}(\mathrm{M})$ \\
\hline & & 1474 & 0.21 & 0.04 & $\sigma \mathrm{CH}_{2}(\mathrm{P})$ \\
\hline \multirow[t]{3}{*}{1442 vs } & $1441 \mathrm{~m}$ & 1472 & 0.02 & 0.04 & $\sigma \mathrm{CH}_{2}(\mathrm{~A})$ \\
\hline & & 1468 & 0.66 & 0.10 & $\sigma \mathrm{CH}_{2}(\mathrm{M})+v \mathrm{CN}(\mathrm{T})$ \\
\hline & & 1466 & 0.01 & 0.01 & $\sigma \mathrm{CH}_{2}(\mathrm{~A})$ \\
\hline \multirow[t]{2}{*}{$1418 \mathrm{~m}$} & & 1465 & 0.26 & 0.01 & $\sigma \mathrm{CH}_{2}(\mathrm{~A})+\sigma \mathrm{CH}_{2}(\mathrm{M})+\sigma \mathrm{CH}_{3}(\mathrm{M})$ \\
\hline & & 1460 & 0.01 & 0.02 & $v \mathrm{CN}(\mathrm{T})+\sigma \mathrm{CH}_{2}(\mathrm{M})+\sigma \mathrm{CH}_{2}(\mathrm{P})$ \\
\hline \multirow[t]{6}{*}{$1404 \mathrm{w}$} & $1404 \mathrm{vw}$ & 1459 & 0.11 & 0.02 & $\sigma \mathrm{CH}_{2}(\mathrm{M})+\sigma \mathrm{CH}_{2}(\mathrm{P})$ \\
\hline & & 1457 & 0.15 & 0.04 & $v \mathrm{CN}(\mathrm{T})+\sigma \mathrm{CH}_{2}(\mathrm{M})$ \\
\hline & & 1455 & 0.30 & 0.08 & $\sigma \mathrm{CH}_{2}(\mathrm{~A})+\sigma \mathrm{CH}_{2}(\mathrm{M})$ \\
\hline & & 1451 & 0.05 & 0.05 & $\sigma \mathrm{CH}_{2}(\mathrm{~A})$ \\
\hline & & 1449 & 0.13 & 0.04 & $\sigma \mathrm{CH}_{2}(\mathrm{P})+\sigma \mathrm{CH}_{2}(\mathrm{M})+v \mathrm{CN}(\mathrm{T})$ \\
\hline & & 1445 & 0.01 & 0.04 & $\sigma \mathrm{CH}_{2}(\mathrm{~A})+\sigma \mathrm{CH}_{2}(\mathrm{M})$ \\
\hline \multirow[t]{2}{*}{$1383 \mathrm{sh}$} & $1384 \mathrm{w}$ & 1426 & 0.05 & 0.01 & $v \mathrm{CC}(\mathrm{P})+\sigma \mathrm{CH}_{2}(\mathrm{M})+\sigma \mathrm{CH}_{2}(\mathrm{P})$ \\
\hline & & 1417 & 0.07 & 0.06 & $v \mathrm{CN}(\mathrm{T})+\sigma \mathrm{CH}_{2}(\mathrm{M})$ \\
\hline \multirow[t]{5}{*}{$1377 \mathrm{~s}$} & $1374 \mathrm{w}$ & 1410 & 0.09 & 0.03 & $\sigma \mathrm{CH}_{2}(\mathrm{P})+\sigma \mathrm{CH}_{2}(\mathrm{M})$ \\
\hline & & 1401 & 0.54 & 0.03 & $v \mathrm{CN}(\mathrm{T})+\sigma \mathrm{CH}_{2}(\mathrm{M})$ \\
\hline & & 1397 & 0.05 & 0.02 & $v \mathrm{CC}(\mathrm{M})+\sigma \mathrm{CH}_{2}(\mathrm{M})$ \\
\hline & & 1395 & 0.03 & 0.01 & $v \mathrm{CC}(\mathrm{A})+\sigma \mathrm{C}_{2} \mathrm{H}(\mathrm{A})$ \\
\hline & & 1391 & 0.01 & 0.01 & $v \mathrm{CC}(\mathrm{A})+\sigma \mathrm{C}_{2} \mathrm{H}(\mathrm{A})$ \\
\hline \multirow[t]{2}{*}{$1362 \mathrm{~s}$} & $1361 \mathrm{sh}$ & 1390 & 0.20 & 0.02 & $\omega \mathrm{CH}_{2}(\mathrm{M})+\omega \mathrm{CH}_{2}(\mathrm{P})$ \\
\hline & & 1385 & 0.04 & 0.01 & $\omega \mathrm{CH}_{2}(\mathrm{~A})$ \\
\hline $1356 \mathrm{sh}$ & & 1380 & 0.33 & 0.01 & $v \mathrm{CN}(\mathrm{T})+\omega \mathrm{CH}_{2}(\mathrm{M})+\omega \mathrm{CH}_{3}(\mathrm{M})$ \\
\hline \multirow[t]{4}{*}{$1344 \mathrm{sh}$} & & 1376 & 0.00 & 0.04 & $\omega \mathrm{CHN}(\mathrm{P})$ \\
\hline & & 1374 & 0.00 & 0.00 & $\omega \mathrm{CH}_{2}(\mathrm{~A})$ \\
\hline & & 1372 & 0.01 & 0.00 & $\omega \mathrm{CH}_{2}(\mathrm{~A})$ \\
\hline & & 1363 & 0.03 & 0.00 & $v \mathrm{CC}(\mathrm{Ph})+\omega \mathrm{CH}_{2}(\mathrm{P})$ \\
\hline \multirow[t]{3}{*}{$1335 \mathrm{~s}$} & 1338 vs & 1355 & 0.64 & 0.03 & $v \mathrm{C}(\mathrm{M}) \mathrm{N}(\mathrm{T})+\sigma \mathrm{CNN}(\mathrm{T})$ \\
\hline & & 1351 & 0.25 & 0.03 & $v \mathrm{CC}(\mathrm{Ph})+\omega \mathrm{CH}_{2}(\mathrm{P})$ \\
\hline & & 1348 & 0.07 & 0.01 & $v \mathrm{CC}(\mathrm{A})$ \\
\hline \multirow[t]{4}{*}{1321} & & 1343 & 0.02 & 0.00 & $\omega \mathrm{CH}_{2}(\mathrm{P})$ \\
\hline & & 1340 & 0.05 & 0.01 & $\omega \mathrm{CH}_{2}(\mathrm{~A})$ \\
\hline & & 1338 & 0.01 & 0.01 & $v \mathrm{CC}(\mathrm{Ph})+v \mathrm{CN}(\mathrm{P})$ \\
\hline & $1311 \mathrm{vs}$ & 1337 & 0.05 & 0.00 & $v \mathrm{CC}(\mathrm{A})+\omega \mathrm{CH}_{2}(\mathrm{~A})$ \\
\hline \multirow[t]{3}{*}{$1296 \mathrm{w}$} & $1301 \mathrm{vs}$ & 1334 & 0.13 & 0.01 & $v \mathrm{CN}(\mathrm{T})+v \mathrm{CN}(\mathrm{P})$ \\
\hline & & 1328 & 0.09 & 0.03 & $\sigma \mathrm{NCH}(\mathrm{P})$ \\
\hline & & 1317 & 0.18 & 0.00 & $v \mathrm{CN}(\mathrm{T})+v \mathrm{CC}(\mathrm{A})$ \\
\hline $1279 \mathrm{~m}$ & & 1314 & 0.01 & 0.00 & $v \mathrm{CC}(\mathrm{A})+\gamma \mathrm{CH}_{2}(\mathrm{~A})$ \\
\hline
\end{tabular}


TABLE 1. (Continued)

\begin{tabular}{|c|c|c|c|c|c|}
\hline$v_{\mathrm{IR}}^{\exp }$ & $v_{\text {Raman }}^{\exp }$ & $v^{\text {calc }}$ & $I_{\mathrm{IR}}^{\mathrm{calc}}$ & $I_{\text {Raman }}^{\text {calc }}$ & Assignment \\
\hline \multirow{7}{*}{$1254 \mathrm{w}$} & \multirow{6}{*}{$1257 \mathrm{w}$} & 1312 & 0.00 & 0.00 & $v \mathrm{CC}(\mathrm{A})$ \\
\hline & & 1310 & 0.36 & 0.01 & $\gamma \mathrm{CH}_{2}(\mathrm{M})+v \mathrm{CN}(\mathrm{T})+v \mathrm{CN}(\mathrm{P})$ \\
\hline & & 1282 & 0.10 & 0.04 & $v \mathrm{CN}(\mathrm{T})+v \mathrm{CS}+v \mathrm{CC}(\mathrm{A})$ \\
\hline & & 1277 & 0.12 & 0.02 & $v \mathrm{CC}(\mathrm{A})+\gamma \mathrm{CH}_{2}(\mathrm{M})$ \\
\hline & & 1275 & 0.01 & 0.06 & $\gamma \mathrm{CH}_{2}(\mathrm{~A})$ \\
\hline & & 1270 & 0.02 & 0.02 & $\sigma \mathrm{NCN}(\mathrm{T})$ \\
\hline & $1243 \mathrm{w}$ & 1254 & 1.00 & 0.04 & $v \mathrm{CC}(\mathrm{Ph})+v \mathrm{CN}(\mathrm{P})$ \\
\hline $1224 \mathrm{~s}$ & $1225 \mathrm{vw}$ & 1235 & 0.11 & 0.02 & $\omega \mathrm{CH}_{2}(\mathrm{M})+\omega \mathrm{CH}_{2}(\mathrm{P})$ \\
\hline $1211 \mathrm{sh}$ & & 1221 & 0.03 & 0.03 & $\omega \mathrm{CH}_{2}(\mathrm{P})$ \\
\hline $1201 \mathrm{w}$ & & 1205 & 0.01 & 0.05 & $\gamma \mathrm{CH}_{2}(\mathrm{~A})$ \\
\hline \multirow[t]{2}{*}{$1190 \mathrm{w}$} & & 1202 & 0.00 & 0.05 & $\gamma \mathrm{CH}_{2}(\mathrm{~A})$ \\
\hline & & 1201 & 0.02 & 0.02 & $\omega \mathrm{CCH}(\mathrm{Ph})$ \\
\hline $1176 \mathrm{w}$ & $1178 \mathrm{~m}$ & 1191 & 0.06 & 0.01 & $v \mathrm{CN}(\mathrm{T})+v \mathrm{CN}(\mathrm{P})$ \\
\hline $1153 \mathrm{~s}$ & $1156 \mathrm{vw}$ & 1174 & 0.16 & 0.03 & $v \mathrm{CN}(\mathrm{P})$ \\
\hline \multirow[t]{6}{*}{$1147 \mathrm{~s}$} & $1143 \mathrm{vw}$ & 1172 & 0.46 & 0.04 & $v \mathrm{C}(\mathrm{M}) \mathrm{N}(\mathrm{P})$ \\
\hline & & 1170 & 0.01 & 0.02 & $\omega \mathrm{CCH}(\mathrm{Ph})$ \\
\hline & & 1138 & 0.00 & 0.00 & $\omega \mathrm{CH}_{2}(\mathrm{~A})$ \\
\hline & & 1134 & 0.35 & 0.00 & $v \mathrm{CN}(\mathrm{T})+\omega \mathrm{CH}_{2}(\mathrm{M})$ \\
\hline & & 1130 & 0.01 & 0.00 & $\gamma \mathrm{CH}_{2}(\mathrm{~A})$ \\
\hline & & 1124 & 0.04 & 0.01 & $\rho \mathrm{CH}_{2}(\mathrm{~A})$ \\
\hline \multirow[t]{2}{*}{$1109 \mathrm{~m}$} & & 1120 & 0.02 & 0.02 & $\rho \mathrm{CH}_{2}(\mathrm{~A})$ \\
\hline & & 1118 & 0.01 & 0.01 & $\rho \mathrm{CH}_{2}(\mathrm{~A})$ \\
\hline \multirow[t]{2}{*}{$1100 \mathrm{~s}$} & $1099 \mathrm{~m}$ & 1108 & 0.07 & 0.02 & $v \mathrm{CN}(\mathrm{P})$ \\
\hline & & 1106 & 0.02 & 0.00 & $v \mathrm{CC}(\mathrm{Ph})$ \\
\hline \multirow[t]{4}{*}{$1084 \mathrm{sh}$} & & 1102 & 0.02 & 0.01 & $\omega \mathrm{CH}_{2}(\mathrm{M})$ \\
\hline & & 1079 & 0.01 & 0.01 & $\omega \mathrm{CH}_{2}(\mathrm{P})$ \\
\hline & & 1072 & 0.00 & 0.04 & $\omega \mathrm{CH}_{2}(\mathrm{P})$ \\
\hline & & 1070 & 0.05 & 0.02 & $v \mathrm{CN}(\mathrm{T})+v \mathrm{CN}(\mathrm{P})$ \\
\hline 1048 & $1051 \mathrm{vw}$ & 1066 & 0.06 & 0.00 & $v \mathrm{CN}(\mathrm{T})+v \mathrm{CC}(\mathrm{A})$ \\
\hline \multirow[t]{3}{*}{$1043 \mathrm{sh}$} & & 1057 & 0.00 & 0.00 & $v \mathrm{CC}(\mathrm{A})$ \\
\hline & $1034 \mathrm{w}$. & 1055 & 0.04 & 0.08 & $v \mathrm{CC}(\mathrm{Ph})$ \\
\hline & & 1054 & 0.00 & 0.00 & $v \mathrm{CC}(\mathrm{A})$ \\
\hline $1026 \mathrm{vw}$ & & 1052 & 0.00 & 0.00 & $v \mathrm{CC}(\mathrm{A})$ \\
\hline \multirow[t]{2}{*}{$1006 \mathrm{~s}$} & & 1021 & 0.39 & 0.01 & $v \mathrm{CN}(\mathrm{P})+\omega \mathrm{CH}_{2}(\mathrm{M})$ \\
\hline & & 1015 & 0.08 & 0.00 & $v \mathrm{CN}(\mathrm{T})+\omega \mathrm{CH}_{2}(\mathrm{M})$ \\
\hline $996 \mathrm{~m}$ & 993 vs & 1003 & 0.01 & 0.12 & $\delta \mathrm{CCC}(\mathrm{Ph})$ \\
\hline \multirow[t]{5}{*}{$983 \mathrm{vw}$} & & 994 & 0.01 & 0.00 & $v \mathrm{CC}(\mathrm{Ph})+v \mathrm{CH}(\mathrm{Ph})$ \\
\hline & & 994 & 0.02 & 0.03 & $v \mathrm{CC}(\mathrm{A})$ \\
\hline & & 986 & 0.00 & 0.03 & $v \mathrm{CC}(\mathrm{A})$ \\
\hline & & 983 & 0.00 & 0.02 & $v \mathrm{CC}(\mathrm{A})$ \\
\hline & & 977 & 0.05 & 0.01 & $v \mathrm{CN}(\mathrm{T})+\omega \mathrm{CH}_{2}(\mathrm{M})$ \\
\hline \multirow[t]{2}{*}{$963 \mathrm{~m}$} & $966 \mathrm{~m}$ & 973 & 0.00 & 0.00 & $v \mathrm{CC}(\mathrm{Ph})+v \mathrm{CH}(\mathrm{Ph})$ \\
\hline & & 961 & 0.09 & 0.00 & $v \mathrm{CN}(\mathrm{P})+\omega \mathrm{CH}_{2}(\mathrm{M})$ \\
\hline
\end{tabular}


TABLE 1. (Continued)

\begin{tabular}{|c|c|c|c|c|c|}
\hline$v_{\mathrm{IR}}^{\exp }$ & $v_{\text {Raman }}^{\exp }$ & $v^{\text {calc }}$ & $I_{\mathrm{IR}}^{\text {calc }}$ & $I_{\text {Raman }}^{\text {calc }}$ & Assignment \\
\hline $943 \mathrm{w}$ & & 951 & 0.00 & 0.01 & $v \mathrm{CC}(\mathrm{A})$ \\
\hline $934 \mathrm{~m}$ & $934 \mathrm{vw}$ & 948 & 0.00 & 0.00 & $v \mathrm{CC}(\mathrm{A})$ \\
\hline $923 \mathrm{~m}$ & $921 \mathrm{vw}$ & 943 & 0.11 & 0.01 & $v \mathrm{CC}(\mathrm{Ph})+v \mathrm{CN}(\mathrm{P})$ \\
\hline \multirow[t]{4}{*}{897 w } & & 900 & 0.02 & 0.00 & $v \mathrm{CC}(\mathrm{Ph})+v \mathrm{CH}(\mathrm{Ph})$ \\
\hline & & 896 & 0.00 & 0.00 & $\rho \mathrm{CH}_{2}(\mathrm{~A})$ \\
\hline & & 890 & 0.00 & 0.00 & $\rho \mathrm{CH}_{2}(\mathrm{~A})$ \\
\hline & & 884 & 0.00 & 0.00 & $\rho \mathrm{CH}_{2}(\mathrm{~A})$ \\
\hline \multirow[t]{4}{*}{$865 \mathrm{w}$} & $868 \mathrm{vw}$ & 881 & 0.03 & 0.02 & $v \mathrm{CC}(\mathrm{A})$ \\
\hline & & 861 & 0.01 & 0.00 & $\rho \mathrm{CH}_{2}(\mathrm{P})$ \\
\hline & & 837 & 0.01 & 0.01 & $\rho \mathrm{CH}(\mathrm{Ph})$ \\
\hline & & 827 & 0.00 & 0.00 & $v \mathrm{CC}(\mathrm{A})$ \\
\hline \multirow[t]{2}{*}{$813 \mathrm{w}$} & $809 \mathrm{vw}$ & 824 & 0.01 & 0.00 & $v \mathrm{CC}(\mathrm{A})$ \\
\hline & & 819 & 0.05 & 0.04 & $v \mathrm{CN}(\mathrm{P})+v \mathrm{C}(\mathrm{M}) \mathrm{N}(\mathrm{P})$ \\
\hline $798 \mathrm{w}$ & & 807 & 0.07 & 0.01 & $v \mathrm{C}(\mathrm{M}) \mathrm{N}(\mathrm{T})+\delta$ (o.o.p. $) \mathrm{CNC}(\mathrm{T})$ \\
\hline $779 \mathrm{~s}$ & & 779 & 0.21 & 0.00 & $\rho \mathrm{CH}_{2}(\mathrm{M})$ \\
\hline 766 vs & $763 \mathrm{~m}$ & 775 & 0.12 & 0.01 & $\delta($ o.o.p. $) \mathrm{CH}(\mathrm{Ph})+\delta \mathrm{CCC}(\mathrm{A})$ \\
\hline \multirow[t]{2}{*}{$743 \mathrm{w}$} & & 770 & 0.08 & 0.07 & $\delta \mathrm{CCC}(\mathrm{A})$ \\
\hline & $734 \mathrm{w}$ & 747 & 0.02 & 0.03 & $\delta($ i.p. $) \mathrm{CCC}(\mathrm{Ph})$ \\
\hline $721 \mathrm{w}$ & & 728 & 0.00 & 0.00 & $\omega \mathrm{CN}_{2}(\mathrm{~T})$ \\
\hline $696 \mathrm{~s}$ & $699 \mathrm{vw}$ & 710 & 0.12 & 0.00 & $\delta($ o.o.p. $) \mathrm{CCC}(\mathrm{Ph})$ \\
\hline \multirow[t]{2}{*}{$675 \mathrm{~m}$} & $675 \mathrm{vw}$ & 688 & 0.01 & 0.02 & $\delta \mathrm{CCC}(\mathrm{A})+\omega \mathrm{CN}_{2}(\mathrm{~T})$ \\
\hline & & 683 & 0.02 & 0.00 & $\delta$ (o.o.p.)CNC(T) \\
\hline \multirow[t]{21}{*}{$663 \mathrm{~m}$} & $661 \mathrm{vw}$ & 669 & 0.01 & 0.02 & $\delta($ o.o.p. $) \mathrm{CNC}(\mathrm{T})$ \\
\hline & $640 \mathrm{vw}$ & 656 & 0.00 & 0.00 & $\delta \mathrm{CCC}(\mathrm{A})$ \\
\hline & & 655 & 0.00 & 0.01 & $\delta \mathrm{CCC}(\mathrm{A})$ \\
\hline & & 645 & 0.00 & 0.01 & $\delta$ (i.p.) $\mathrm{CCC}(\mathrm{Ph})+\rho \mathrm{CH}_{2}(\mathrm{P})$ \\
\hline & $614 \mathrm{vw}$ & 629 & 0.00 & 0.01 & $\delta($ i.p. $) \mathrm{CCC}(\mathrm{Ph})$ \\
\hline & $594 \mathrm{vw}$ & 604 & 0.02 & 0.01 & $\delta$ (i.p.) $\mathrm{CNC}(\mathrm{T})+\gamma \mathrm{CH}_{2}(\mathrm{M})$ \\
\hline & $542 \mathrm{vw}$ & 551 & 0.03 & 0.01 & $v \mathrm{CS}+\rho \mathrm{CH}_{2}(\mathrm{M})+\rho \mathrm{CH}_{2}(\mathrm{P})$ \\
\hline & & 549 & 0.01 & 0.01 & $v \mathrm{CS}+\rho \mathrm{CH}_{2}(\mathrm{M})+\rho \mathrm{CH}_{2}(\mathrm{P})$ \\
\hline & $525 \mathrm{vw}$ & 536 & 0.05 & 0.00 & $\delta$ (i.p.) $\mathrm{CCC}(\mathrm{Ph})+\rho \mathrm{CH}_{2}(\mathrm{P})$ \\
\hline & $496 \mathrm{vw}$ & 493 & 0.00 & 0.00 & $\delta \mathrm{CNC}(\mathrm{P})$ \\
\hline & $465 \mathrm{vw}$ & 468 & 0.01 & 0.00 & $\delta \mathrm{CCC}(\mathrm{A})$ \\
\hline & & 461 & 0.01 & 0.00 & $\delta \mathrm{CCC}(\mathrm{A})+\omega \mathrm{CN}_{2}(\mathrm{~T})$ \\
\hline & & 451 & 0.00 & 0.01 & $\delta \mathrm{CCC}(\mathrm{A})+\delta \mathrm{CNC}(\mathrm{P})+\delta \mathrm{CH}_{2}(\mathrm{M})$ \\
\hline & $444 \mathrm{vw}$ & 446 & 0.01 & 0.01 & $\delta \mathrm{CCC}(\mathrm{A})+\delta \mathrm{CNC}(\mathrm{P})+\delta \mathrm{CH}_{2}(\mathrm{M})$ \\
\hline & & 431 & 0.01 & 0.00 & $\delta \mathrm{CCC}(\mathrm{A})+\delta \mathrm{CNC}(\mathrm{T})$ \\
\hline & & 428 & 0.01 & 0.00 & $\delta \mathrm{CNC}(\mathrm{P})+\delta \mathrm{CH}_{2}(\mathrm{M})+\delta \mathrm{CNC}(\mathrm{T})$ \\
\hline & & 420 & 0.01 & 0.00 & $\delta($ o.o.p. $) \mathrm{CCC}(\mathrm{Ph})$ \\
\hline & $409 \mathrm{vw}$ & 411 & 0.00 & 0.00 & $\delta \mathrm{CCC}(\mathrm{A})+\delta \mathrm{CNC}(\mathrm{P})$ \\
\hline & & 411 & 0.00 & 0.00 & $\delta \mathrm{CCC}(\mathrm{A})+\delta \mathrm{CNC}(\mathrm{P})$ \\
\hline & & 408 & 0.00 & 0.00 & $\delta \mathrm{CCC}(\mathrm{A})$ \\
\hline & $362 \mathrm{vw}$ & 364 & 0.00 & 0.00 & $\delta \mathrm{CCC}(\mathrm{A})$ \\
\hline
\end{tabular}


TABLE 1. (Continued)

\begin{tabular}{|c|c|c|c|c|c|}
\hline$v_{\mathrm{IR}}^{\exp }$ & $v_{\text {Raman }}^{\exp }$ & $v^{\text {calc }}$ & $I_{\mathrm{IR}}^{\text {calc }}$ & $I_{\text {Raman }}^{\text {calc }}$ & Assignment \\
\hline & & 359 & 0.02 & 0.01 & $\delta \mathrm{CCC}(\mathrm{A})+\delta \mathrm{CNC}(\mathrm{P})+\delta \mathrm{CH}_{2}(\mathrm{M})$ \\
\hline & & 342 & 0.02 & 0.00 & $\delta \mathrm{CCC}(\mathrm{Ph})+\delta \mathrm{CNC}(\mathrm{P})+\delta \mathrm{CH}_{2}(\mathrm{M})$ \\
\hline & & 323 & 0.03 & 0.00 & $\delta \mathrm{CNC}(\mathrm{P})$ \\
\hline & $316 \mathrm{vw}$ & 317 & 0.00 & 0.00 & $\delta \mathrm{CCC}(\mathrm{A})$ \\
\hline & $305 \mathrm{vw}$ & 314 & 0.00 & 0.00 & $\delta($ o.o.p. $) \mathrm{CNC}(\mathrm{T})+\sigma \mathrm{HCC}(\mathrm{M})$ \\
\hline & & 303 & 0.00 & 0.00 & $\delta$ (o.o.p.) CNC(T) $+\sigma \mathrm{HCC}(\mathrm{M})+\delta \mathrm{CNC}(\mathrm{P})$ \\
\hline & $272 \mathrm{vw}$ & 276 & 0.01 & 0.00 & $v \mathrm{CS}+\rho \mathrm{CH}_{2}(\mathrm{P})+\delta \mathrm{CCC}(\mathrm{Ph})$ \\
\hline & & 264 & 0.01 & 0.00 & $v \mathrm{CS}+\rho \mathrm{CH}_{2}(\mathrm{M})+\rho \mathrm{CH}_{2}(\mathrm{P})$ \\
\hline & & 250 & 0.01 & 0.00 & $\rho \mathrm{CH}_{2}(\mathrm{M})+\rho \mathrm{CH}_{2}(\mathrm{P})$ \\
\hline & $245 \mathrm{vw}$ & 248 & 0.01 & 0.00 & $\rho \mathrm{CH}_{2}(\mathrm{M})+\rho \mathrm{CH}_{3}(\mathrm{M})$ \\
\hline & & 231 & 0.01 & 0.01 & $\rho \mathrm{CSN}(\mathrm{T})+\rho \mathrm{CH}_{2}(\mathrm{P})+\delta \mathrm{CCC}(\mathrm{Ph})$ \\
\hline & $207 \mathrm{vw}$ & 209 & 0.01 & 0.01 & $\rho \mathrm{CSN}(\mathrm{T})+\rho \mathrm{CH}_{2}(\mathrm{P})+\delta \mathrm{CCC}(\mathrm{Ph})$ \\
\hline & & 200 & 0.00 & 0.00 & $\rho \mathrm{CSN}(\mathrm{T})+\rho \mathrm{CH}_{2}(\mathrm{P})+\delta \mathrm{CCC}(\mathrm{Ph})+\delta \mathrm{CCC}(\mathrm{A})$ \\
\hline & $160 \mathrm{w}$ & 186 & 0.01 & 0.00 & $\delta \mathrm{CC}(\mathrm{Et}) \mathrm{N}(\mathrm{T})$ \\
\hline
\end{tabular}

Note. Band and line intensities $I_{\mathrm{IR}}$ and $I_{\mathrm{Raman}}$ are normalized to the strongest band or line; vs, very strong; s, strong; m, medium; w, weak; vw, very weak; sh, shoulder; normal modes: $v$, stretching; $\delta$, bending; $\rho$, rocking; $\sigma$, scissoring; $\omega$, wagging; $\gamma$, twisting; i.p., in-plane bending; o.o.p., out-of-plane bending vibrations.

(e.g., adamantyl, phenyl, or ethyl) possessed highly characteristic vibrational frequencies that, as a rule, did not mix with vibrations of other functional groups. The other fragments, e.g., triazole, methyl, and piperazine, could in several instances be considered a molecular framework so that the vibrations of these fragments mixed and were delocalized over the whole framework.

The calculated structural parameters of the isolated molecule gave deviations of $\leq 1 \%$ for most calculated bond lengths from the experimental ones. Only bonds involving the $\mathrm{N}$ atoms (i.e., $\mathrm{C}-\mathrm{N}$ and $\mathrm{N}-\mathrm{N}$ of the piperazine and triazole rings) had errors of $\sim 2 \%$ for the calculated bond lengths. A similar situation was observed previously for $N^{\prime}$-(adamantan2-ylidene)benzohydrazide [21], 4,4'-methylenediphenyldiisocyanate [38], and benzohydrazide [39] and was most probably characteristic of the used combination of basis set and functional. An analogous tendency was found for the in-plane angles. Angles between $\mathrm{C}-\mathrm{N}$ bonds typically had the greatest errors (up to 4\%). In other instances, the deviations of the calculated values from the experimental ones were usually $\leq 1 \%$. It is also noteworthy that the $\mathrm{C}-\mathrm{H}$ bond lengths that were fixed in the x-ray structure analysis [19] at $0.97 \AA(\mathrm{A}, \mathrm{P}, \mathrm{M}), 0.96(\mathrm{Et})$, and $0.93(\mathrm{Ph})$ differed noticeably from the experimental values for adamantane (1.112 $\pm 0.004 \AA[40])$, phenol (1.080-1.086, average $1.083 \AA$ [41]), piperazine (1.133 $\AA$ [42]), and methylene (1.085 $\AA$ [43]).

Figure 2 shows the experimental and calculated IR and Raman spectra of the studied compound. Table 1 presents experimental vibrational frequencies and those calculated at the B3LYP/cc-pVDZ level of theory and the corresponding intensities in the IR and Raman spectra. In most instances, the vibrations were localized on one of the functional groups (adamantyl, phenyl, ethyl, piperazine, methylene, or triazole).

Vibrations of the adamantyl fragment. Frequencies of $\mathrm{CH}$ - and $\mathrm{CH}_{2}$-stretching vibrations in monosubstituted adamantyl lay in the range $2952-2850 \mathrm{~cm}^{-1}$ [44]. According to the literature [44] and our calculations [20, 22], bands at 2943, 2934, 2911, 2907, 2888, and $2953 \mathrm{~cm}^{-1}$ in the IR spectrum and lines at 2941, 2917, 2909, 2889, 2855, and $2847 \mathrm{~cm}^{-1}$ in the Raman spectrum belonged to adamantyl $\mathrm{CH}$ and $\mathrm{CH}_{2}$ stretching vibrations. Adamantyl $\mathrm{CH}_{2}$ scissoring vibrations were located at $1475-1440 \mathrm{~cm}^{-1}$ [44]. Monosubstitution lifted the degeneracy of $\mathrm{CH}_{2}$ wagging vibrations $\left(\sim 1350 \mathrm{~cm}^{-1}\right)$ and led to the appearance of a specific sequence of bands in the range 1400-1300 $\mathrm{cm}^{-1}$ [45]. Thus, bands at $\sim 1472$ and $1441 \mathrm{~cm}^{-1}$ were assigned to scissoring vibrations of adamantane $\mathrm{CH}_{2}$ groups; bands and lines at 1362,1321 , and $1311 \mathrm{~cm}^{-1}$, to wagging vibrations. Furthermore, adamantyl vibrations appeared near $1200\left(\mathrm{CH}_{2}\right.$ twisting vibrations $), 1100\left(\mathrm{CH}_{2}\right.$ rocking vibrations $)$, 800 (CC stretching vibrations) and in the ranges $780-740$ and $460-330 \mathrm{~cm}^{-1}$ (adamantyl CCC bending vibrations) [44, 45]. Thus, bands at 1201 and $1190 \mathrm{~cm}^{-1}$ corresponded to $\mathrm{CH}_{2}$ twisting vibrations; bands and lines at 865 (IR), 868 (Raman), 
813 (IR), and $809 \mathrm{~cm}^{-1}$ (Raman), to CC stretching vibrations; and 743 (IR), 640 (Raman), 465 (Raman), 362 (Raman) and $316 \mathrm{~cm}^{-1}$ (Raman), to CCC bending vibrations.

Vibrations of the phenyl group. Stretching vibrations of $\mathrm{CH}-$ and CC-bonds in aromatic compounds are usually found in the ranges $3100-3000$ and $1630-1200 \mathrm{~cm}^{-1}$ [46]. Thus, the spectrum of benzene has bands at $\sim 1596,1486$, and $1310 \mathrm{~cm}^{-1}$ [47]. This allowed us to assign the bands at 1599, 1579, 1495, and $1450 \mathrm{~cm}^{-1}$ in the IR spectrum and lines at 1602 and $1453 \mathrm{~cm}^{-1}$ in the Raman spectrum to phenyl-ring CC stretching vibrations. Strong IR bands at 996 and $969 \mathrm{~cm}^{-1}$ and a Raman line at $993 \mathrm{~cm}^{-1}$ were attributed to ring bending vibrations (1010 and $707 \mathrm{~cm}^{-1}$ in the spectrum of benzene [47]). In-plane and out-of-plane ring bending vibrations were also observed near 734 and $614 \mathrm{~cm}^{-1}$.

Vibrations of ethyl and methyl groups. $\mathrm{CH}_{2}$ and $\mathrm{CH}_{3}$ stretching vibrations were observed at 2980-2950 $\mathrm{cm}^{-1}$ [46]. Bands at 2977 and $2971 \mathrm{~cm}^{-1}$ in the IR spectrum and lines at 2970 and $2953 \mathrm{~cm}^{-1}$ in the Raman spectrum were assigned to stretching vibrations of these groups. Twisting and wagging vibrations lay in the range $1370-1080 \mathrm{~cm}^{-1}$ [46]; scissoring and rocking vibrations, $\sim 1080$ and $780 \mathrm{~cm}^{-1}$ [46]. It is noteworthy that methylene vibrations were mixed with bending vibrations of triazole and piperazine rings linked to it.

Vibrations of the piperazine group. According to the literature [42, 48], $\mathrm{CH}_{2}$ stretching vibrations in pure piperazine appeared at 3087, 2987, 2914, 2853, and 2750 (IR [42]); 2833 and 2771 (Raman [42]); and $2944 \mathrm{~cm}^{-1}$ (IR [48]). In our opinion, frequencies of 2750 and $2771 \mathrm{~cm}^{-1}$ are too low for $\mathrm{CH}_{2}$ stretching vibrations and the aforementioned bands and lines were more likely combined frequencies or overtones (Table 1). Thus, the band at $2826 \mathrm{~cm}^{-1}$ in the IR spectrum of the studied molecule was assigned to piperazine-ring $\mathrm{CH}$ and $\mathrm{CH}_{2}$ stretching vibrations. Scissoring and wagging $\mathrm{CH}_{2}$ vibrations for pure piperazine [42, 48] and 1-phenylpiperazine [49] were observed at 1458-1431 [48], 1426 [42], 1407 [49], 1390, 1364, 1360 [42], and $1190 \mathrm{~cm}^{-1}$ [49]. Bands and lines at 1377,1321 , and $1211 \mathrm{~cm}^{-1}$ were attributed to $\sigma \mathrm{CH}_{2}-$ and $\omega \mathrm{CH}_{2}$ vibrations of the piperazine ring. Stretching vibrations of heteroaromatic rings were found to interact with each other [46]. Therefore, it was impossible to isolate vibrational frequencies of separate CC-, CN-, or NN-bonds. Stretching vibrations of heteroaromatic rings were observed at $1600-1300 \mathrm{~cm}^{-1}$ [46], i.e., in the same region as for aromatic rings, and were often masked by each other. Bands at 1177, 1115, and $933 \mathrm{~cm}^{-1}$ in the IR spectrum and lines at 1184, 1109, and $836 \mathrm{~cm}^{-1}$ in the Raman spectrum of pure piperazine were identified as belonging to ring vibrations [48]. Researchers also assigned bands and lines for pure piperazine at 1323 (IR), 1294 (Raman), 1268 (IR), 1218 (IR), 1173 (IR), 1120 (Raman), 1055 (IR), and $1049 \mathrm{~cm}^{-1}$ (Raman) to CN stretching vibrations; a band at $1199 \mathrm{~cm}^{-1}$ in the IR spectrum and a line at $1186 \mathrm{~cm}^{-1}$ in the Raman spectrum, to CC stretching vibrations [42]. Thus, according to the literature [42, 48] and our calculations, bands at 1153,1100, and $1006 \mathrm{~cm}^{-1}$ in the IR spectrum and 1156 and $1099 \mathrm{~cm}^{-1}$ in the Raman spectrum were assigned to piperazine skeletal (CC and CN bonds) stretching vibrations. In-plane and out-of-plane piperazine-ring bending vibrations lay below $920 \mathrm{~cm}^{-1}$. The corresponding spectral bands and lines were assigned based on our calculations.

Vibrations of the triazole ring. The situation was analogous for skeletal stretching vibrations of the triazole ring. $\mathrm{CN}$, $\mathrm{NN}$, and CS stretching vibrations coupled with each other. Their frequencies depended on the type of substitution. Breathing vibrations of the $1 H-1,2,4$-triazole ring were observed at 1540 [50], 1495-1482 [50, 51], 1473 [50], 1390-1361 [50, 51], 1272-1255 [51], 1146 [51], 1190 [50], and 1062-1055 $\mathrm{cm}^{-1}$ [51]. Thus, bands and lines near 1533 (IR), 1529 (Raman), 1338 (Raman), 1335 (IR), 1257 (Raman), 1254 (IR), 1051 (Raman) and $1048 \mathrm{~cm}^{-1}$ (IR) belonged to triazole-ring breathing vibrations. Other triazole-ring bending vibrations lay below $800 \mathrm{~cm}^{-1}$. The corresponding bands and lines were also assigned based on our calculations. Bands at 1418,1383, 1356, 1296, 1224, 1176, and $1147 \mathrm{~cm}^{-1}$ in the IR spectrum and lines at 1384, $1301,1243,1225,1178$, and $1143 \mathrm{~cm}^{-1}$ in the Raman spectrum corresponded according to our calculations to stretching and bending vibrations delocalized over various functional groups of the molecular framework.

UV/Vis spectrum. Figure 3 (curve 1) shows the experimental electronic absorption spectrum of the studied compound dissolved in EtOH. The long-wavelength absorption band maximum was located at $252 \mathrm{~nm}$. The possibility of a bathochromic shift of the band due to the influence of the polar solvent $(\mathrm{EtOH})$ and the fact that the purely electronic $0-0$-transition (the wavelength of which was also calculated quantum chemically) lay on the long-wavelength side of the absorption band had to be considered in order to compare correctly the quantum-chemically calculated and experimental electronic absorption spectra. On this basis, it could be assumed that the wavelength of the $0-0$-transition of the pure compound should coincide approximately with the wavelength of the absorption-band maximum in EtOH, i.e., in the region 250-255 nm.

Figure 3 and Table 2 also show electronic absorption spectra calculated in the approximations TDDFT/CAM-B3LYP/ cc-pVDZ, TDDFT/CAM-B3LYP/cc-pVDZ+SMD (EtOH), and CASSCF(2,4)/XMCQDPT2. These results indicated that the calculations using TDDFT and the CAM-factor were unsatisfactory for describing the observed electronic absorption spectra. Transitions into lower electronic states $\left(S_{1} \leftarrow S_{0}, S_{2} \leftarrow S_{0}\right)$ had practically zero intensity. The $S_{1} \leftarrow S_{0}$-transition experienced 


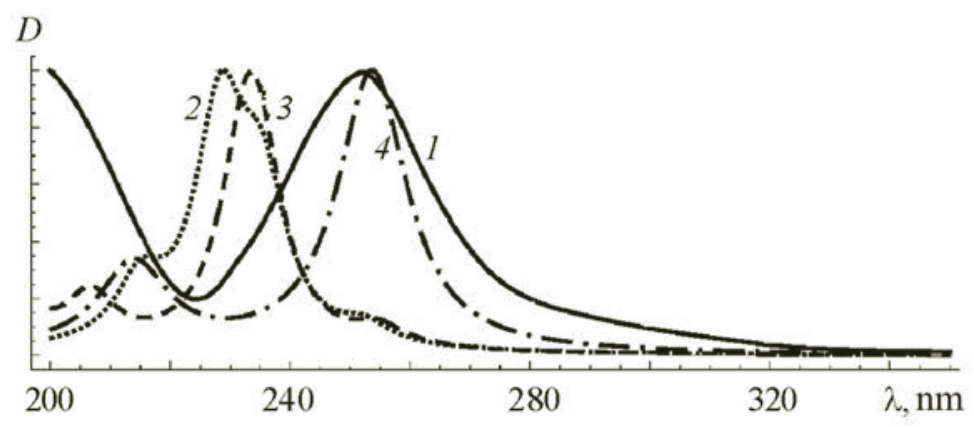

Fig. 3. Experimental (1) and calculated [TDDFT/CAM-B3LYP/cc-pVDZ (2); TDDFT/CAM-B3LYP/cc-pVDZ+SMD (EtOH) (3); and CASSCF(2,4)/XMCQDPT2

(4)] electronic absorption spectra of the studied compound.

TABLE 2. Bond Lengths $(\lambda)$ and Oscillator Strengths $(f)$ of Electronic Transitions of 3-(Adamantan-1-yl)-4-ethyl-1[(4-phenylpiperazin-1-yl)methyl]-1H-1,2,4-triazole-5(4H)-thione Calculated at Various Theory Levels

\begin{tabular}{|c|c|c|c|c|c|c|}
\hline \multirow{2}{*}{ Transition } & \multicolumn{3}{|c|}{ TDDFT } & \multicolumn{2}{c|}{ CASSCF(2,4)/XMCQDPT2 } \\
\cline { 2 - 6 } & \multicolumn{2}{|c|}{ CAM-B3LY/cc-pVDZ } & \multicolumn{2}{c|}{ CAM-B3LYP/cc-pVDZ+ SMD (EtOH) } & \multicolumn{2}{c|}{} \\
\cline { 2 - 6 } & $\lambda, \mathrm{nm}$ & $f$ & $\lambda, \mathrm{nm}$ & $f$ & $\lambda, \mathrm{nm}$ & $f$ \\
\hline$S_{1} \leftarrow S_{0}$ & 272 & 0.001 & 263 & 0.003 & 214 & 0.795 \\
$S_{2} \leftarrow S_{0}$ & 252 & 0.028 & 255 & 0.045 & 156 & 0.374 \\
$S_{3} \leftarrow S_{0}$ & 235 & 0.215 & 235 & 0.283 & 101 & 0.001 \\
$S_{4} \leftarrow S_{0}$ & 228 & 0.337 & 232 & 0.411 & \\
\hline
\end{tabular}

TABLE 3. Greatest Biological Activities of 3-(Adamantan-1-yl)-4-ethyl-1-[(4-phenylpiperazin-1-yl)methyl]-1H-1,2,4-triazole5(4H)-thione Predicted Based on Compound Structure

\begin{tabular}{|c|c|c|}
\hline$P a$ & $P i$ & Activity \\
\hline 0.590 & 0.037 & Proteasome ATP-ase inhibitor \\
0.496 & 0.003 & $11 \beta$-Hydroxysteroid dehydrogenase inhibitor \\
0.487 & 0.044 & Analgesic \\
0.459 & 0.047 & Polarization stimulator \\
0.438 & 0.008 & $\alpha$-Adrenoreceptor antagonist \\
0.412 & 0.067 & Muramoyltetrapeptide carboxypeptidase inhibitor \\
\hline
\end{tabular}

a hypsochromic shift of $9 \mathrm{~nm}$ if the solvent was considered. It was supposed that errors in the TDDFT calculations were responsible for the ICT in the studied compound.

Figure 4 shows the upper "frozen" (doubly occupied) and four active molecular orbitals (MOs) from the total active space and also their occupancy factors (second, third, and fourth active MOs had the same occupancy factor). Figure 4 shows that the electron density shifted upon excitation partially from the piperazine and phenyl groups to the triazole ring although it was mostly localized on the S atom. Thus, the phenyl and piperazine rings in the studied compound acted as electron-density donors; the triazole, as an acceptor.

The wavelength of the $S_{1} \leftarrow S_{0}$-transition that was calculated in the CASSCF(2,4)/XMCQDPT2 approximation [34] was $254 \mathrm{~nm}$. The transition had a high intensity (Table 2; Fig. 3, curve 4). Thus, the electronic absorption spectrum of the studied compound that was calculated in terms of the multi-reference perturbation theory method agreed fully with the experimental data. 


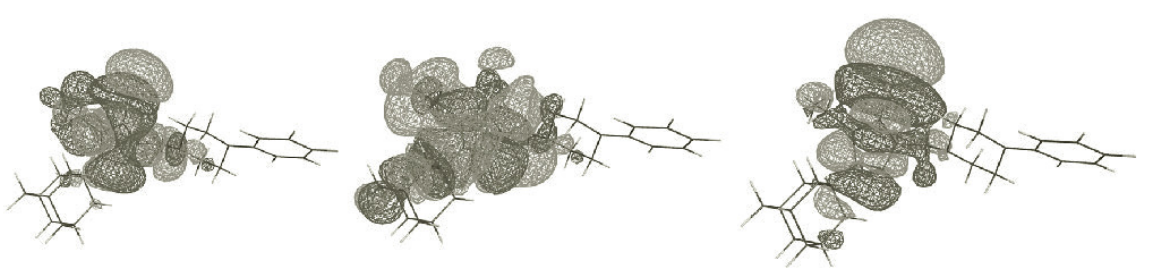

Second, third, and fourth active MOs (0.286)

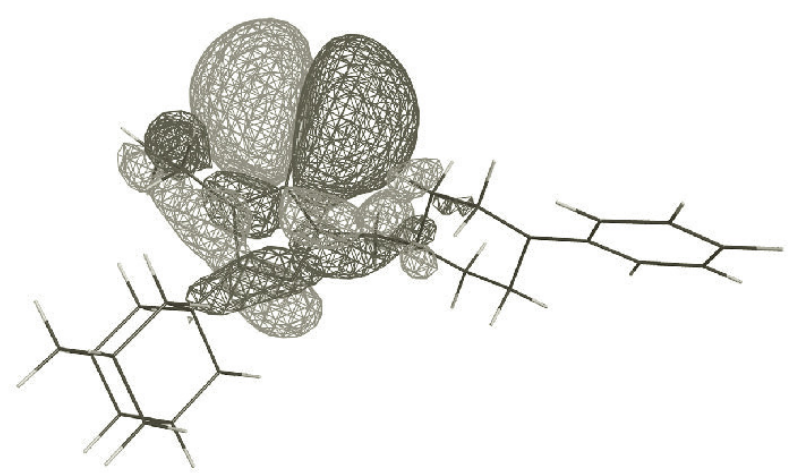

First active MO (1.142)

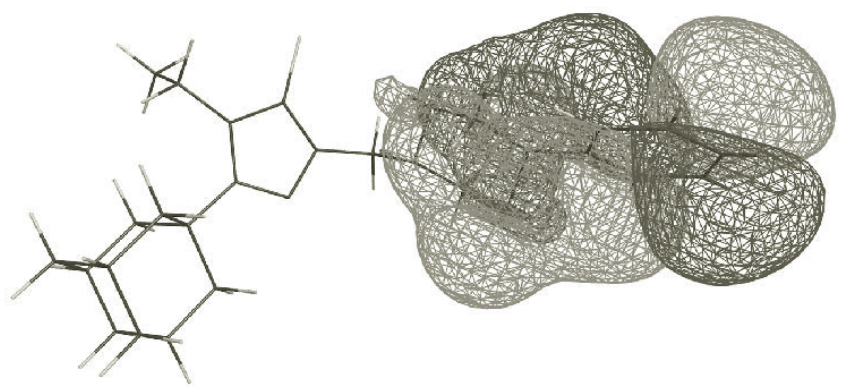

Second doubly occupied MO (2.000)

Fig. 4. Higher occupied MO, four active MOs, and their occupancy factors calculated in the CASSCF $(2,4)$ approximation.

Biological activities. Table 3 presents the biological activity indices of the studied compound (i.e., the probability of activity $P a$ or inactivity $P i$ of the determined type) that were found using the PASS Online database [37]. According to the literature [36], if $P a>0.7$, then the examined compound will most probably demonstrate this activity experimentally. If $0.5<P a<0.7$, then the compound may demonstrate experimental activity but the probability of this is less. If $P a<0.5$, the likelihood of this compound demonstrating this activity is low. According to Table 3, the studied compound probably acted as a proteasome ATP-ase inhibitor.

Conclusions. Spectral characteristics of 3-(adamantan-1-yl)-4-ethyl-1-[(4-phenylpiperazin-1-yl)methyl]-1H1,2,4-triazole-5(4H)-thione, an adamantane-containing compound that is promising for drug development, were analyzed systematically. Vibrational IR and Raman spectra of the crystalline compound were interpreted based on quantum-chemical simulation in the B3LYP/cc-pVDZ approximation. Biological activity indices of the molecule were predicted based on the molecular structure and established that the studied compound was likely to act as a proteasome ATP-ase inhibitor. Simulation of the UV/Vis absorption spectrum using TDDFT and $a b$ initio perturbation theory demonstrated that the first approximation was inadequate for describing the experimental spectrum of the studied compound. This was most likely a consequent of ICT. The calculations in the second of the aforementioned approximations agreed with the experimental data. The results could be used in medicinal chemistry and for analytical purposes.

Acknowledgment. The work was financially supported in part by the Deanship of Scientific Research at Princess Nourah bint Abdulrahman University through the Research Group Program (No. RGP-1438-0010). 


\section{REFERENCES}

1. G. Lamoureux and G. Artavia, Curr. Med. Chem., 17, 2967-2978 (2010).

2. J. Liu, D. Obando, V. Liao, T. Lifa, and R. Codd, Eur. J. Med. Chem., 46, 1949-1963 (2011).

3. G. Ali Mansoori, P. L. Barros de Araujo, and E. Silvano de Araujo, Diamondoid Molecules: With Applications in Biomedicine, Materials Science, Nanotechnology \& Petroleum Science, World Scientific Publishing (2012).

4. F. G. Hayden, Antiviral Res., 71, 372-378 (2006).

5. M.-J. Perez-Perez, J. Balzarini, M. Hosoya, E. De Clercq, and M.-J. Camarasa, Bioorg. Med. Chem. Lett., 2, 647-648 (1992).

6. I. Stylianakis, A. Kolocouris, N. Kolocouris, G. Fytas, G. B. Foscolos, E. Padalko, J. Neyts, and E. De Clercq, Bioorg. Med. Chem. Lett., 13, 1699-1703 (2003).

7. N. A. Ilyushina, N. V. Bovin, R. G. Webster, and E. A. Govorkova, Antiviral Res., 70, 121-131 (2006).

8. G. Zoidis, C. Fytas, I. Papanastasiou, G.B. Foscolos, G. Fytas, E. Padalko, E. De Clercq, L. Naesens, J. Neyts, and N. Kolocouris, Bioorg. Med. Chem., 14, 3341-3348 (2006)

9. A. A. El-Emam, O. A. Al-Deeb, M. A. Al-Omar, and J. Lehmann, Bioorg. Med. Chem., 12, 5107-5113 (2004).

10. M. Protopopova, C. Hanrahan, B. Nikonenko, R. Samala, P. Chen, J. Gearhart, L. Einck, and C. A. Nacy, J. Antimicrob. Chemother., 56, 968-974 (2005).

11. A. A. El-Emam, A.-M. S. Al-Tamimi, M. A. Al-Omar, K. A. Alrashood, and E. E. Habib, Eur. J. Med. Chem., 68, 96-102 (2013).

12. A. A. Kadi, E. S. Al-Abdullah, I. A. Shehata, E. E. Habib, T. M. Ibrahim, and A. A. El-Emam, Eur. J. Med. Chem., 45, 5006-5011 (2010).

13. K. Omar, A. Geronikaki, P. Zoumpoulakis, C. Camoutsis, M. Sokovic, A. Ciric, and J. Glamoclija, Bioorg. Med. Chem., 18, 426-432 (2010).

14. A. A. Kadi, N. R. El-Brollosy, O. A. Al-Deeb, E. E. Habib, T. M. Ibrahim, and A. A. El-Emam, Eur. J. Med. Chem., 42, 235-242 (2007).

15. E. S. Al-Abdullah, H. H. Asiri, S. Lahsasni, E. E. Habib, T. M. Ibrahim, and A. A. El-Emam, Drug Des., Dev. Ther., 8, 505-518 (2014).

16. O. Kouatly, A. Geronikaki, C. Kamoutsis, D. Hadjipavlou-Litina, and P. Eleftheriou, Eur. J. Med. Chem., 44, 1198-1204 (2009).

17. O. A. Al-Deeb, M. A. Al-Omar, N. R. El-Brollosy, E. E. Habib, T. M. Ibrahim, and A. A. El-Emam, Arzneim. Forsch./Drug Res., 56, 40-47 (2006).

18. S. Riganas, I. Papanastasiou, G. B. Foscolos, A. Tsotinis, J.-J. Bourguignon, G. Serin, J.-F. Mirjolet, K. Dimas, V. N. Kourafalos, A. Eleutheriades, V. I. Moutsos, H. Khan, S. Georgakopoulou, A. Zaniou, M. Prassa, M. Theodoropoulou, S. Pondiki, and A. Vamvakides, Bioorg. Med. Chem., 20, 3323-3331 (2012).

19. A. A. El-Emam, E. S. Al-Abdullah, H. M. Al-Tuwaijri, M. Said-Abdelbaky, and S. Garcia-Granda, Acta Crystallogr., Sect. E: Struct. Rep. Online, 68, o2380-o2381 (2012).

20. M. B. Shundalau, E. S. Al-Abdullah, E. V. Shabunya-Klyachkovskaya, A. V. Hlinisty, O. A. Al-Deeb, A. A. El-Emam, and S. V. Gaponenko, J. Mol. Struct., 1115, 258-266 (2016).

21. A. M. Andrianov, I. A. Kashyn, V. M. Andrianov, M. B. Shundalau, A. V. Hlinisty, S. V. Gaponenko, E. V. ShabunyaKlyachkovskaya, A. Matsukovich, A.-M. S. Al-Tamimi, and A. A. El-Emam, J. Chem. Sci., 128, 1933-1942 (2016).

22. M. Shundalau, Y. Mindarava, A. Matsukovich, S. Gaponenko, and A. A. El-Emam, in: Abstracts of the XIVth International Conference on Molecular Spectroscopy (ICMS2017), Bialka Tatrzanska, Poland (2017), p. 136.

23. M. W. Schmidt, K. K. Baldridge, J. A. Boatz, S. T. Elbert, M. S. Gordon, J. H. Jensen, S. Koseki, N. Matsunaga, K. A. Nguyen, S. J. Su, T. L. Windus, M. Dupuis, and J. A. Montgomery, Jr., J. Comput. Chem., 14, 1347-1363 (1993).

24. B. M. Bode and M. S. Gordon, J. Mol. Graphics Modell., 16, 133-138 (1998).

25. L. J. Farrugia, J. Appl. Crystallogr, 30, 565 (1997).

26. T. H. Dunning, Jr., J. Chem. Phys., 90, 1007-1023 (1989).

27. A. D. Becke, J. Chem. Phys., 98, 5648-5652 (1993).

28. C. Lee, W. Yang, and R. G. Parr, Phys. Rev. B: Condens. Matter Mater. Phys., 37, 785-789 (1988).

29. P. J. Stephens, F. J. Devlin, C. F. Chabalowski, and M. J. Frisch, J. Phys. Chem., 98, 11623-11627 (1994).

30. E. Runge and E. K. U. Gross, Phys. Rev. Lett., 52, 997-1000 (1984).

31. K. Burke, J. Werschnik, and E. K. U. Gross, J. Chem. Phys., 123, 062206 (2005). 
32. T. Yanai, D. P. Tew, and N. C. Handy, Chem. Phys. Lett., 393, 51-57 (2004).

33. A. V. Marenich, C. J. Cramer, and D. G. Truhlar, J. Phys. Chem. B, 113, 6378-6396 (2009).

34. A. A. Granovsky, J. Chem. Phys., 134, 214113 (2011).

35. Alex A. Granovsky, Firefly version 8 ; http://classic.chem.msu.su/gran/firefly/index.html.

36. A. Lagunin, A. Stepanchikova, D. Filimonov, and V. Poroikov, Bioinformatics, 16, 747-748 (2000).

37. PASS Online; http://way2drug.com/passonline/index.php.

38. G. A. Pitsevich, M. B. Shundalau, M. A. Ksenofontov, and D. S. Umreiko, Global J. Anal. Chem., 2, 114-124 (2011).

39. V. Arjunan, T. Rani, C. V. Mythili, and S. Mohan, Spectrochim. Acta, Part A, 79, 486-496 (2011).

40. I. Hargittai and K. Hedberg, in: Molecular Structures and Vibrations, S. J. Cyvin (Ed.), Elsevier Publishing Co., New York (1972), pp. 340-357.

41. N. W. Larsen, J. Mol. Struct., 51, 175-190 (1979).

42. S. Gunasekaran and B. Anita, Indian J. Pure Appl. Phys., 46, 833-838 (2008).

43. K. Kuchitsu (Ed.), Structure of Free Polyatomic Molecules. Basic Data, Springer (1998).

44. L. Bistricic, G. Baranovic, and K. Mlinaric-Majerski, Spectrochim. Acta, Part A, 51, 1643-1664 (1995).

45. E. I. Bagrii, Adamantanes: Preparation, Properties, Applications [in Russian], Nauka, Moscow (1989).

46. R. M. Silverstein, F. X. Webster, and D. J. Kiemle, Spectrometric Identification of Organic Compounds, 7th edn., Wiley \& Sons, Inc., (2005).

47. L. Goodman, A. G. Ozkabak, and S. N. Thakur, J. Phys. Chem., 95, 9044-9058 (1991).

48. P. J. Hendra and D. B. Powell, Spectrochim. Acta, 18, 299-306 (1962).

49. O. Alver, C. Parlak, and M. Şenyel, Spectrochim. Acta, Part A, 67, 793-801 (2007).

50. S. A. Kudchadker and C. N. R. Rao, Indian J. Chem., 11, 140-142 (1973).

51. F. Billes, H. Endredi, and G. Keresztury, J. Mol. Struct.: THEOCHEM, 530, 183-200 (2000). 